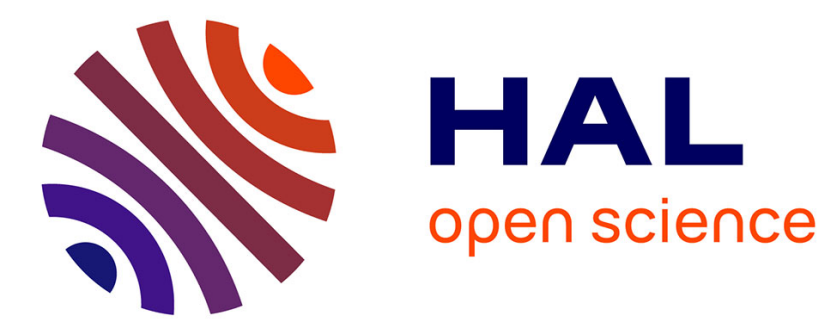

\title{
MODIFICATION DE LA FRÉQUENCE FONDAMENTALE DE LA VOIX DES PILOTES : INCIDENTS RÉELS ET SIMULÉS
}

B. Gramatica, R. Ruiz, C. Legros

\section{- To cite this version:}

B. Gramatica, R. Ruiz, C. Legros. MODIFICATION DE LA FRÉQUENCE FONDAMENTALE DE LA VOIX DES PILOTES : INCIDENTS RÉELS ET SIMULÉS. Journal de Physique IV Proceedings, 1992, 02 (C1), pp.C1-335-C1-338. 10.1051/jp4:1992172 • jpa-00251244

\section{HAL Id: jpa-00251244 \\ https://hal.science/jpa-00251244}

Submitted on 1 Jan 1992

HAL is a multi-disciplinary open access archive for the deposit and dissemination of scientific research documents, whether they are published or not. The documents may come from teaching and research institutions in France or abroad, or from public or private research centers.
L'archive ouverte pluridisciplinaire HAL, est destinée au dépôt et à la diffusion de documents scientifiques de niveau recherche, publiés ou non, émanant des établissements d'enseignement et de recherche français ou étrangers, des laboratoires publics ou privés. 


\title{
MODIFICATION DE LA FREQUENCE FONDAMENTALE DE LA VOIX DES PILOTES : INCIDENTS RÉELS ET SIMULÉS
}

\author{
B. GRAMATICA, R. RUIZ et C. LEGROS
}

Laboratoire d'Acoustique de Métrologie d'Instrumentation, Université Paul Sabatier, 38 rue des 36 Ponts, F-31400 Toulouse cedex, France

\section{Résumé:}

Un message vocal, outre son contenu sémantique, véhicule des informations sur l'état psycho-physiologique du locuteur. La fatigue physique, le stress psychologique sont des éléments pathologiques de cet état. Un indicateur acoustique connu comme sensible pour la manifestation vocale d'un "stress" est la fréquence fondamentale $F_{0}$. Dans cette étude, nous avons travaillé sur ce paramètre de manière macroscopique sur des moyennes de $F_{0}$ et de manière plus fine en effectuant un suivi temporel de $F_{0}$. Les supports sont de deux types: un enregistrement en simulateur et l'enregistreur phonique d'un avion détruit.

\section{Abstract:}

A vocal message, apart from its semantic content, carries information about the psychological and physiological condition of a speaker. Physical fatigue and psychological stress are pathological elements of the condition. A well-known indicator of the vocal manifestation of the stress is the fundamental frequency $F_{0}$. In this study, we worked on this parameter, first with mean values, afterwards by following up its time representation. Two records were analysed, the first in a flight simulator, the second being the Cockpit Voice Recorder of a destroyed airplane.

\section{INTRODUCTION}

L'apparition d'un incident de vol à bord d'un aéronef entraîne des modifications de l'état émotionnel des pilotes. Le trouble, la gêne, l'inquiétude, ou plus généralement le "stress" que ressentent les personnels navigants peut altérer leur expression vocale, en particulier, la fréquence fondamentale. Ses variations sont successivement étudiées pour des incidents de vol simulés (enregistrement en simulateur d'hélicoptère) et pour un incident réél grâce à l'analyse de l'enregistreur phonique (Cockpit Voice Recorder) d'un aéronef détruit. On a pu remarquer dans un cas comme dans l'autre, des modifications notables de la fréquence fondamentale, pour les incidents inhabituels ou fortuits en simulateur et pour la phase finale du vol ainsi qu'à certaines apparitions de l'incident dans le cas réel. 


\section{PRESENTATION DE L'ETUDE}

L'étude de la variation de la fréquence fondamentale n'est pas nouvelle. En effet, de nombreux travaux ont été publiés sur le sujet. Ces études concluent toutes à une augmentation de la fréquence fondamentale avec la charge de travail, qu'elle soit réelle $[1,2]$ ou simulée $[1,3,4]$. Nous calculons la fréquence fondamentale des voyelles extraites à l'aide du logiciel de traitement du signal ILS, et avons fixé la fréquence d'échantillonnage à $10 \mathrm{kHz}$. La méthode utilisée par ce logiciel pour la détection et la mesure de $F_{0}$ est une méthode cepstrale.

\section{EXPERIMENTATION EN SIMULATEUR}

Pour cette première partie de l'étude, nous avons analysé la voix d'un élève-pilote, d'une base aérienne, en simulateur d'hélicoptère de type PUMA. L'enregistrement, d'une durée de 1 H 30 minutes a été effectué avec des microphones AKG type C140/B, de sensibilité $20 \mathrm{mV} / \mathrm{Pa}$, de bande passante $150-10000 \mathrm{~Hz}$.

L'enregistreur était un magnétophone numérique SONY de type DAT. Sur cette bande, près de 600 phonèmes ont été extraits. 83 d'entre eux caractérisent la période dite de repos qui servira de référence d'un état émotionnel neutre. 99 sont tirés de l'indicatif de l'appareil (COTAM 2.35), ce qui permet un suivi sur un même groupe de phonèmes de l'évolution de $F_{0}$. Les autres sont extraits des périodes précédant les incidents et des périodes suivant la constatation du problème. Pour chacun des incidents, provoqués par l'instructeur ou fortuits (erreurs de changement de fréquence radio), ainsi que pour la période de repos, la fréquence fondamentale moyenne et son écart-type associé ont été calculés, pour les phonèmes prononcés avant et après chaque panne. Ces résultats sont consignés dans le tableau 1.

Tableau 1 : Fréquence fondamentale moyenne avant et après chaque incident avant : entre parenthèses; après : sans parenthèses

\begin{tabular}{|c|c|c|c|c|c|c|c|c|}
\hline & Repos & Train & $\begin{array}{c}\text { Fréq. } \\
\text { radio }\end{array}$ & Mode C & $\begin{array}{c}\text { Pitot } \\
\text { droit }\end{array}$ & $\begin{array}{c}\text { Givrage } \\
\text { turbine }\end{array}$ & $\begin{array}{c}\text { Pompe de } \\
\text { gavage }\end{array}$ & $\begin{array}{c}\text { Transfo. } \\
\text { redresseur }\end{array}$ \\
\hline$F_{0}(H z)$ & 117 & 112 & $\mathbf{1 4 5}$ & 118 & 107 & $\mathbf{1 2 2}$ & $\mathbf{1 2 3}$ & 114.8 \\
& & $(121)$ & & $(126)$ & $(115)$ & $(\mathbf{1 2 5})$ & $(\mathbf{1 3 1})$ & $(118)$ \\
$\sigma(H z)$ & 11.4 & 14 & $\mathbf{1 0}$ & 2 & 5.7 & $\mathbf{2 5}$ & $\mathbf{1 1 . 5}$ & 8.8 \\
& & $(21.8)$ & & $(14.5)$ & $(23.8)$ & $(\mathbf{1 9})$ & $(\mathbf{1 2 . 4})$ & $(9.4)$ \\
\hline
\end{tabular}

\begin{tabular}{|c|c|c|c|c|c|c|c|}
\hline & $\begin{array}{l}\text { Fréq. } \\
\text { radio }\end{array}$ & $\begin{array}{l}\text { Fausse } \\
\text { alarme } \\
\text { turb. } 1\end{array}$ & $\begin{array}{c}\text { Niveau } \\
\text { hydraulique } \\
\text { droit }\end{array}$ & $\begin{array}{l}\text { Panne } \\
\text { radio- } \\
\text { altimètre }\end{array}$ & $\begin{array}{c}\text { Niveau } \\
\text { hydraulique } \\
\text { droit }\end{array}$ & Glide & $\begin{array}{l}\text { Panne } \\
\text { moteur }\end{array}$ \\
\hline$F_{0}(H z)$ & 142 & $\begin{array}{c}122 \\
(123)\end{array}$ & $\begin{array}{c}113 \\
(122)\end{array}$ & 147.8 & $\begin{array}{c}120 \\
(127)\end{array}$ & $\begin{array}{r}130.7 \\
(123.5)\end{array}$ & $\begin{array}{c}136.7 \\
(131.5)\end{array}$ \\
\hline$\sigma(H z)$ & 15.5 & $\begin{array}{c}10 \\
(13)\end{array}$ & $\begin{array}{c}10.6 \\
(15.6)\end{array}$ & 18.9 & $\begin{array}{c}12 \\
(8)\end{array}$ & $\begin{array}{c}16.3 \\
(10.26)\end{array}$ & $\begin{array}{c}16.2 \\
(13.2)\end{array}$ \\
\hline
\end{tabular}


On peut observer à l'aide d'un test de Student qu'un certain nombre d'incidents provoquent une augmentation significative de la fréquence fondamentale par rapport à la période de repos (en gras dans le tableau). Cette variation n'est toutefois pas confirmée par rapport au périodes précédant les incidents. On peut quand même observer une augmentation de $F_{0}$ (/avant) pour les incidents de fin de vol ainsi que pour les pannes fortuites que constituent les erreurs de changement de fréquence. Dûes à l'inattention du pilote, elles ne sont pas des pannes habituelles du simulateur. Relativement entraîné, ce pilote présente peu de perturbations. L'intensité émotionnelle est incomparable avec celle engendrée par un vol réel.

Toutefois, il est important de constater que les pannes inhabituelles au simulateur ainsi que l'accumulation d'incidents graves en fin de vol, provoquent une réaction notable chez cet individu, constatée par de fortes variations de $F_{0}$ en fonction du temps. Leur dynamique est de $106 \mathrm{~Hz}$ soit une variation relative par rapport à la $F_{0}$ moyenne en période de repos de $90 \%$. Cette valeur est certes remarquable, mais très inférieure à celle obtenue en situation réelle.

\section{CAS REEL}

Le cas réel que nous avons étudié est l'analyse de l'enregistreur phonique d'un aéronef ayant connu une panne grave affectant les commandes de vol à deux reprises. La deuxième a été fatale. Cet enregistrement nous a été communiqué par le Bureau Enquète Accident en contrepartie d'un engagement de stricte confidentialité assorti d'un droit de regard sur son utilisation. Le pilote place gauche (pilote $\mathrm{G}$ ) était aux commandes, le pilote place droite (pilote $D$ ) avait une position hiérarchique supérieure. Là aussi 600 phonèmes ont été extraits du discours du pilote $\mathrm{G}$ et du pilote $\mathrm{D}$ au cours des 30 dernières minutes du vol. Les fréquences fondamentales moyennes accompagnées de leur écart type pour chaque phase du vol sont consignées dans le tableau suivant.

Tableau 2: Fréquence fondamentale moyenne par phases

\begin{tabular}{|c|c|c|c|c|c|c|}
\hline Phase & 1 & 2 & 3 & 4 & 5 & 6 \\
\hline$F_{0}$ pilote $G$ & 144 & 107 & 151 & 149 & 158 & 154 \\
\hline$\sigma$ pilote $G$ & 26 & 10 & 33 & 31 & 45 & 45 \\
\hline$F_{0}$ pilote $D$ & 136 & 156 & 150 & 157 & 146 & 221 \\
\hline$\sigma$ pilote $D$ & 21 & 24 & 19 & 19 & 27 & 81 \\
\hline
\end{tabular}

Un test de Student n'a pas permis de mettre en évidence la significativité des variations de la fréquence fondamentale pour chaque phase du vol par rapport à une période que l'on peut considérer comme émotionnellement neutre, à savoir le roulage et la préparation au décollage (3). Par contre, un suivi plus fin de la fréquence fondamentale a ensuite été mené et a permis de relever des fluctuations anormales de $200 \mathrm{~Hz}$ soit $132 \%$ pour le pilote $\mathrm{G}$ et de $264 \mathrm{~Hz}$ soit $175 \%$ pour le pilote D par rapport à la $F_{0}$ au repos. 
Cependant, lors de cette analyse, on peut s'apercevoir que la réaction du locuteur est retardée par rapport aux instants où apparaissent les incidents techniques.

Le pilote $\mathrm{G}$ tente de résoudre le problème et sa réaction vocale ne se manifeste qu'après l'ouverture de la discussion qui suit la constatation de la panne. Les deux membres d'équipage n'ont pas la même réaction émotionnelle face aux incidents. Le pilote D (supérieur hiérarchique) aura pour attitude de rassurer son collègue ; il gardera un calme certain jusqu'à la phase ultime du vol, ce qui est confirmé par les faibles fluctuations de $F_{0}$ hormis durant cette dernière période. Ce suivi temporel nous montre aussi que la réaction à un stimulus perturbateur peut être ponctuelle ou étalée dans le temps.

\section{$\mathrm{V}$ CONCLUSION}

Le cas réel a apporté des variations plus probantes de la fréquence fondamentale. On pourrait donc envisager la poursuite de l'étude non plus en simulateur mais en phase d'évaluation en vol ce qui entraînerait chez les pilotes des réactions émotionnelles plus flagrantes et permettrait la reproductibilité des conditions expérimentales. L'analyse des résultats obtenus dans les deux cas nous a permis de confirmer que la fréquence fondamentale est une caractéristique acoustique sensible aux manifestations du trouble émotionnel engendré par l'apparition d'incidents de vol.

\section{REMERCIEMENTS}

Nous avons pu mener cette étude grâce à la collaboration de l'Armée de l'Air (Base de Francazal) qui nous a aimablement permis l'accès à ses simulateurs de vol.

Nous remercions également le Bureau Enquêtes-Accident qui nous a permis de disposer d'extraits d'enregistrement.

\section{BIBLIOGRAPHIE}

[1] RuIz R., Contribution à l'étude d'une charge de travail sur les caractéristiques acoustiques de la voix, Thèse de doctorat de l'université Paul Sabatier, Toulouse (1991).

[2] Williams C.E., Stevens K.N., On determining the emotional state of pilots during flight: an exploratory study, Aerospace Med.,40(12),1369-1372, (December 1969).

[3] Ruiz R., Legros C., Analyse de la voix de pilotes en situation de stress dans un simulateur de vol, Colloque de Physique C2, supplément au $N^{\circ} 2$,tome 51,(Février 1990), premier congrès français d'Acoustique, Lyon (1990).

[4] Legros C., RuIz R., Etude de l'influence de la charge de travail sur les caractéristiques acoustiques de la voix II, Expérimentations en simulateur, rapport CNES, (Février 1990). 